\title{
BMJ Open Suitability and acceptability of the Carer Support Needs Assessment Tool (CSNAT) for the assessment of carers of people with MND: a qualitative study
}

\author{
Gail Ewing (D) , ${ }^{1}$ Sarah Croke, ${ }^{2}$ Christine Rowland, ${ }^{2}$ Gunn Grande ${ }^{2}$
}

To cite: Ewing G, Croke S, Rowland C, et al. Suitability and acceptability of the Carer Support Needs Assessment Tool (CSNAT) for the assessment of carers of people with MND: a qualitative study. BMJ Open 2020;10:e039031. doi:10.1136/ bmjopen-2020-039031

- Prepublication history for this paper is available online. To view these files, please visit the journal online (http://dx.doi. org/10.1136/bmjopen-2020039031).

Received 02 April 2020 Revised 06 August 2020 Accepted 21 August 2020

Check for updates

(C) Author(s) (or their employer(s)) 2020. Re-use permitted under CC BY-NC. No commercial re-use. See rights and permissions. Published by BMJ.

${ }^{1}$ Centre for Family Research, University of Cambridge,

Cambridge, UK

${ }^{2}$ School of Health Sciences, Faculty of Biology, Medicine and Health, The University of Manchester, Manchester, UK

Correspondence to

Gunn Grande;

gunn.grande@manchester.ac.uk

\section{ABSTRACT}

Objectives Motor neurone disease (MND) is a

progressive, life-limiting illness. Caregiving impacts greatly on family carers with few supportive interventions for carers. We report Stages 1 and 2 of a study to: (1) explore experiences of MND caregiving and use carer-identified support needs to determine suitability and acceptability of the Carer Support Needs Assessment Tool (CSNAT), (2) adapt the CSNAT as necessary for comprehensive assessment and support of MND carers, prior to (Stage 3) feasibility testing.

Design Qualitative: focus groups, interviews and carer workshops.

Setting Three UK MND specialist centres serving a wide range of areas.

Participants Stage 1: 33 carers, 11 from each site: 19 current carers, 14 bereaved. Stage 2: 19 carer advisors: 10 bereaved, 9 current carers. Majority were spouses/ partners ranging in age from under 45 years to over 75 years. Duration of caring: 4 months to 12.5 years.

Results Carers described challenges of a disease that was terminal from the outset, of 'chasing' progressive deterioration, trying to balance normality and patient independence against growing dependence, and intensive involvement in caregiving. Carers had extensive support needs which could be mapped to existing CSNAT domains: both 'enabling' domains which identify carers' needs as co-workers as well as carers' 'direct' needs as clients in relation to their own health and well-being. Only one aspect of their caregiving experience went beyond existing domains: a new domain on support needs with relationship changes was identified to tailor the CSNAT better to MND carers.

Conclusions Carers of people with MND found the adapted CSNAT to be an appropriate and relevant tool for assessment of their support needs. The revised version has potential for assessment of carers in other longer-term caring contexts. A further paper will report the Stage 3 study on feasibility of using the adapted CSNAT in routine practice.

\section{INTRODUCTION}

Motor neurone disease (MND) is a progressive, life-limiting illness that is terminal at diagnosis. Life expectancy is usually between two years to five years, though this can vary
Strengths and limitations of this study

Recruitment of carers from three major motor neurone disease centres in the UK ensured a wide range of caregiving experiences and enhanced transferability of findings.

- Inclusion of both bereaved and current carers enabled reflection on the full duration of caregiving.

- Enrolment of participants as subsequent advisors ensured strong, informed Patient and Public Involvement (PPI) in Carer Support Needs Assessment Tool review and design, and may serve as a pragmatic model for PPI in general.

- The qualitative approach of the study which has a self-selecting sample limits generalisability of the study findings.

in individual cases. The disease is one of progressive muscle weakness affecting movement, speech, swallowing and eventually breathing. In the UK, there are estimated to be 5000 adults living with MND at any one time with six people diagnosed per day, ${ }^{1}$ in Australia about 2000 people are living with MND with two people diagnosed daily ${ }^{2}$ and in the USA, where MND is more commonly known as amyotrophic lateral sclerosis (ALS), up to 16000 adults have ALS with 15 new cases diagnosed each day. ${ }^{3}$

The process of patient deterioration impacts greatly on family members, most often spouses/partners (hereafter referred to as carers), who are the main sources of help and support for patients. Patients often progress to needing long-term assistance with activities of daily living (ADL) such as eating, bathing and toileting which can result in high levels of caregiver burden and a major impact on the physical health and well-being of carers. ${ }^{4-6}$ Managing patients' loss of speech, swallowing and motor function further adds to caring responsibilities and concerns, and to the distress of dealing with a devastating 
disease in a close family member. Unsurprisingly carers of people with MND suffer high levels of psychological morbidity, including anxiety and depression, and have reduced quality of life. ${ }^{7-10}$

Better support has the potential to ameliorate negative impacts of taking on a caregiving role. ${ }^{711}$ However, reviews within the broader palliative care context have not shown such interventions to have major impact on carer outcomes. ${ }^{12-14}$ In an MND context, although reviews show carer well-being to be compromised ${ }^{15}$ carer interventions tested to date have reported limited effects $^{1617}$ and none have been designed specifically for carers of people living with MND. The Carers' Alert Thermometer (CAT) has been used with family carers of people with MND. ${ }^{18}$ The CAT was designed originally as an alert tool in a more general care context and to date it has not undergone testing as a practice intervention in any trial. For MND, its instructions were modified to enable use by Motor Neurone Disease Association (MNDA) volunteers instead of healthcare staff: there was no involvement of carers themselves to review suitability or relevance of the CAT questions prior to its use with MND carers.

To be more effective and provide the support carers need to prevent or reduce negative impacts, interventions must be individually tailored and consider carers' full range of support needs rather than be selective. ${ }^{12}$ Furthermore, they should address support that carers need to manage the carer role to reduce negative impacts (proactive approach), rather than address negative impacts once they occur (reactive approach).

One intervention which has been shown to improve carer support in end-of-life care is the Carer Support Needs Assessment Tool (CSNAT) intervention. The CSNAT intervention enables practitioners to provide comprehensive, person-centred carer assessment and support. For use in practice, the intervention comprises a comprehensive, evidence-based assessment tool ${ }^{19}$ and a defined five-stage person-centred process, ${ }^{20}$ that together allow carers themselves to identify, consider and prioritise their support needs, discuss these with a practitioner and identify supportive input they would find helpful (action plan), with follow-up review. This represents a significant change in practice as support for carers of patients with lifelimiting illnesses is normally informal and unstructured with solutions proposed by practitioners. ${ }^{21}$ The CSNAT has good validity, ${ }^{22}$ the intervention is valued by practitioners and carers, ${ }^{21} 2324$ and improves carer outcomes ${ }^{2526}$ within a palliative home care context. Thus a three-stage study sought to explore the suitability, acceptability and feasibility of the CSNAT intervention in MND caregiving. This paper presents data from the study's first two stages, with objectives (1) to explore the experiences of caregiving in the context of MND and use carer-identified support needs to assess suitability and acceptability of the CSNAT; and (2) to make any adaptions to the existing CSNAT for comprehensive assessment and support of carers of people with MND. A further paper will report
Table 1 Inclusion/exclusion criteria for Stages 1 and 2 recruitment

\begin{tabular}{|c|c|c|}
\hline & Current carers & Bereaved carers \\
\hline Inclusion & $\begin{array}{l}\text { Patient at least } 3 \\
\text { months postdiagnosis }\end{array}$ & $\begin{array}{l}6-12 \text { months } \\
\text { postbereavement }\end{array}$ \\
\hline \multirow[t]{3}{*}{ Exclusion } & Younger than 18 years & Younger than 18 years \\
\hline & $\begin{array}{l}\text { Clinician concerns } \\
\text { about psychological/ } \\
\text { physical ability to cope } \\
\text { with study participation }\end{array}$ & $\begin{array}{l}\text { Clinician concerns about } \\
\text { psychological/physical } \\
\text { ability to cope with study } \\
\text { participation }\end{array}$ \\
\hline & $\begin{array}{l}\text { Unable to give informed } \\
\text { consent }\end{array}$ & $\begin{array}{l}\text { Unable to give informed } \\
\text { consent }\end{array}$ \\
\hline
\end{tabular}

the third stage feasibility study from the perspective of carers and healthcare professionals.

\section{METHODS}

Qualitative design using focus groups (FGs), interviews and workshops involving carers of people with MND.

\section{Setting}

The first two stages of the study were conducted between December 2017 and May 2018 at three MND specialist centres serving patients (and carers) from a wide range of areas. Carers were recruited from all three sites.

All participants provided written consent.

\section{Stage 1: FGs and interviews with carers}

\section{Recruitment}

Sites identified carers from patient databases using purposive sampling to ensure a balance between carer gender, relationship to the patient and type of MND. Both current and bereaved carers were included (see table 1 for inclusion/exclusion criteria). Recruitment was through direct invitation at clinics by the MND consultant/Clinical Nurse Specialist or by postal invitation from the MND consultant.

All carers received a recruitment pack (study invitation letter from the consultant, information leaflet explaining the study, confidentiality of data handling and data protection, reply form and freepost return envelope). Carers interested in taking part responded directly to the study researcher (SC) who provided any further information and made arrangements for data collection.

The three sites identified 170 carers eligible to take part (126 current carers; 44 bereaved). Forty-eight responded to the invitation (28\% response rate); four later withdrew due to worsening patient health. Not all respondents were available to attend a group or interview. In total 33 carers (11 from each site) joined Stage 1. Table 2 summarises participants' characteristics.

\section{Data collection}

Nine FGs were conducted (three at each site), from December 2017 to January 2018, facilitated by two researchers (GE/SC; GE/CR; CR/SC). FGs were chosen 
Table 2 Stage 1 carer participants

\begin{tabular}{ll}
$\begin{array}{l}\text { Bereaved carers } \\
(\mathrm{N}=14)\end{array}$ & $\begin{array}{l}\text { Current carers } \\
(\mathrm{N}=19)\end{array}$ \\
\hline
\end{tabular}

\begin{tabular}{lrr}
\hline \multicolumn{2}{l}{ Relationship to patient } & \\
Spouse/partner & 13 & 17 \\
\hline Daughter/son & 1 & 1 \\
\hline Other & 0 & 1 \\
\hline Age range, years & & \\
$<=45$ & 0 & 2 \\
$46-55$ & 2 & 2 \\
\hline $56-65$ & 1 & 6 \\
$66-75$ & 8 & 6 \\
$>75$ & 3 & 2 \\
\hline Missing & 0 & 1 \\
\hline
\end{tabular}

Carer description of type of MND

\begin{tabular}{lll}
\hline ALS & 5 & 8 \\
\hline MND only & 6 & 1 \\
\hline Bulbar & 3 & 3 \\
\hline $\begin{array}{l}\text { Primary lateral } \\
\text { sclerosis }\end{array}$ & 0 & 2 \\
$\begin{array}{l}\text { Progressive } \\
\text { muscular atrophy }\end{array}$ & 0 & 1 \\
\hline $\begin{array}{l}\text { Not known } \\
\text { Duration of caring }\end{array}$ & 0 & 4 \\
\hline $\begin{array}{l}\text { Less than 1 year } \\
\text { 1-2 years }\end{array}$ & 3 & 1 \\
\hline 3-4 years & 8 & 9 \\
\hline 5-10 years & 1 & 6 \\
\hline More than 10 & 0 & 1 \\
years & & 2 \\
\hline
\end{tabular}

ALS, amyotrophic lateral sclerosis; MND, motor neurone disease.

to enable participants to 'share and compare' experiences, allowing observation of both consensus and diversity of views. ${ }^{27}$ Small groups were held with three to four carers each to maximise discussion: groups averaged $108 \mathrm{~min}$. A distress protocol which identified support contacts at each site was employed to ensure any upset participants were supported. The introduction to the session by the main facilitator recognised the sensitivity of the discussion and assured participants that they could take a break, leave the session or withdraw at any time if they so wished. The confidential nature of the discussion was reiterated. At the end of each session, facilitators checked on whether any participants were upset and that they had contact details for further support if needed. There was a period after each FG where carers could chat and have further refreshments before leaving. None of the participants required additional support as a result of taking part in the FGs/interviews.
The topic guide covered three main areas: (1) a brief introduction about the carer and the person with MND; (2) their experience of key stages of caregiving starting with the time of diagnosis, what was challenging, what help/support they received or would have liked to have had, from whom and when; (3) carers were introduced to the CSNAT intervention and given a copy of the CSNAT (the tool itself) and asked their reaction to the tool and its usefulness to carers of people with MND: anything not relevant; any type of support need missing.

Respite provision was offered to facilitate FG participation. However, where carers felt unable to leave their home, because of caring or other reasons, an individual home interview was conducted by the study researcher (SC) to enable their participation. Four interviews were conducted, each lasting just over an hour, following the same format and topic guide as the FGs. As the interviews were conducted after the majority of FGs were completed, the researcher (SC) was able to share aspects of the FG discussions at individual interviews to have some elements of the 'share and compare' discussion in the groups. As such, there was no substantial difference in the findings between the two approaches. The main benefit of the FG discussions was a personal one of communality of experience and mutual support.

\section{Analysis}

Sessions were audio-recorded and field notes were written. Recordings were fully transcribed, then checked and anonymised by a researcher (SC). Transcripts were read by all researchers for familiarisation. Qualitative content analysis was conducted: $:^{28}$ (1) Conventional content analysis was used to analyse the experience of caregiving in MND allowing codes to emerge from the data to develop an initial coding scheme which was then used to index the data; codes were then clustered into categories. (2) A directed content analysis considered carers' support needs in relation to CSNAT as the tool already provided a framework, mapping data to the existing 14 CSNAT domains. Support needs/supportive input not captured by the CSNAT domain coding scheme were coded separately.

The research team discussed and agreed the coding process which was used by GE to index the transcripts. Atlas.ti was used to facilitate data management. Verification of the indexing process was conducted by a second researcher $(\mathrm{CR})$ and a process of checking and agreeing emergent domains and interpretations was conducted by the entire research team.

\section{Stage 2: Workshops with carer advisors}

\section{Recruitment}

FG/interview participants from Stage 1 were invited to become carer advisors for Stage 2 workshops. Those interested provided contact details to the research team and agreed to further contact.

There were 19 carer advisors: 10 bereaved; 9 current carers. Three of these were carers who had shown interest 
Table 3 Stage 2 carer advisors

\begin{tabular}{ll}
$\begin{array}{l}\text { Bereaved carers } \\
(\mathrm{N}=10)\end{array}$ & $\begin{array}{l}\text { Current carers } \\
(\mathrm{N}=9)\end{array}$ \\
\hline
\end{tabular}

\begin{tabular}{lll}
\hline \multicolumn{2}{l}{ Relationship to patient } \\
Spouse/partner & 9 & 8 \\
\hline Daughter/son & 1 & 0 \\
\hline Other & 0 & 1 \\
\hline Age range, years & & \\
$<=45$ & 0 & 2 \\
$46-55$ & 2 & 1 \\
$56-65$ & 1 & 3 \\
$66-75$ & 5 & 2 \\
$>75$ & 2 & 0 \\
\hline Missing & 0 & 1 \\
\hline
\end{tabular}

Carer description of type of MND

\begin{tabular}{lll}
\hline ALS & 5 & 5 \\
\hline MND only & 3 & 1 \\
\hline Bulbar & 1 & 1 \\
\hline $\begin{array}{l}\text { Primary lateral } \\
\text { sclerosis }\end{array}$ & 0 & 1 \\
$\begin{array}{l}\text { Progressive } \\
\text { muscular atrophy }\end{array}$ & 0 & 0 \\
\hline $\begin{array}{l}\text { Not known } \\
\text { Duration of caring }\end{array}$ & 1 & 1 \\
\hline $\begin{array}{l}\text { Less than 1 year } \\
\text { 1-2 years }\end{array}$ & 3 & 1 \\
\hline 3-4 years & 2 & 4 \\
\hline 5-10 years & 0 & 3 \\
\hline More than 10 & 0 & 1 \\
years & & 0 \\
\hline
\end{tabular}

ALS, amyotrophic lateral sclerosis; MND, motor neurone disease.

in Stage 1 but then were unable to participate at that time. Table 3 summarises the characteristics of the carer advisors.

\section{Data collection}

Three workshops were conducted in May 2018, one at each site. They lasted just under two hours, were facilitated by two researchers (CR/SC) with five to eight carers in each. A workshop guide was used to structure the discussions: (1) a brief background to the study; (2) a reminder about the two-part CSNAT intervention; (3) an overview of Stage 1 findings. Then carer participants were asked to review the findings on the content of the CSNAT: was there anything missing, focusing specifically on any additional domain(s) needed (reported below). Carers' views of the process of using the CSNAT intervention in practice will be reported in a subsequent paper.

At the end of the workshops, 10 participants agreed to help finalise the wording of an additional domain for the CSNAT in the context of MND by email/telephone contact.

Analysis

Workshops were audio-recorded and field notes written. Data processing was the same as Stage 1. As the workshops focused on refining the CSNAT content for the context of MND, directed content analysis using the existing framework of the CSNAT domains was used. At all stages, the coding was shared within the research team, interpretations discussed and agreed.

\section{PATIENT AND PUBLIC INVOLVEMENT}

At the study outset, two researchers (GE and SC) attended a regional MNDA meeting, to introduce the study and have informal discussions with family carers. This led to two follow-up telephone conversations (GE) that provided a wider perspective and understanding of caring for someone with MND, which was used to enhance the sensitivity of subsequent data collection. Additionally, use of carer advisors in Stage 2 provided a strong patient and public involvement element to the CSNAT review and design.

\section{FINDINGS}

The findings are in three main sections: (1) the context of caregiving in MND; (2) the support needs and supportive input derived from the experience of MND caregiving that relate to existing CSNAT domains; and (3) an additional domain of support needs identified within the study. Italics indicate verbatim quotations. To preserve anonymity, participant quotes are identified by alphanumeric codes: the letter $(\mathrm{B})$ indicates the respondent was bereaved and the letter $(\mathrm{C})$ a current carer.

\section{(1) The context of caregiving in MND}

With any life-limiting illness there is a significant emotional impact on the family. MND carers expressed that beyond the 'shock' of diagnosis, they were dealing with an illness that is terminal from the outset: "Well, it is a death sentence, isn't it, [...] but most people with cancer, they've got a little...they've got hope that something...there's very few that actually they get to the stage where it's diagnosed and they say there's absolutely nothing that we can do for you" (BSR017). The great majority of carers in the study were partners/ spouses of the person with MND whose own lives were "on hold" (CSH059) during caregiving. "We've got the illness together" (CSH052) expressed their experience and influenced the support needs they had.

\section{Maintaining normality}

A strong feature in early caregiving was of actively promoting patient independence for as long as possible, to enable patients to retain some normality in the face of their illness. This involved encouraging them to carry on with previous activities, even if this took much longer, for a sense of satisfaction. Tact and diplomacy was often 
required in making adjustments to ADLs to maintain independence. It was hard for carers to know how long to hold back: "It's difficult for him to accept that he is not as active as he used to be. And for me to have the balance between helping where it's needed and not giving help where it's not. [...] How long should I hover there" (CSH041). Carers were keen to avoid 'taking over', enabling patients feel that they were still living a normal life. This had to be tempered with an awareness that some aspects of maintaining independence could also be hazardous. Getting the balance right was an important aspect of early caregiving.

\section{Relationship changes because of MND}

Carers described how the illness and caregiving influenced their relationship with the patient. Patients could be 'stubborn', 'demanding', 'angry'. They fully acknowledged the difficult situation for patients, but certain responses greatly affected carers. As many were couples, there was a changed relationship, for some from the point of diagnosis, with tensions or petty arguments. The disease blurred role boundaries: as husband/wife/ partner and as carer, affecting all aspects of their relationship, particularly when providing personal care. Some talked openly about loss of intimacy due to illness, though others reflected that it was not "top of my list" of concerns (CSR030) as long as closeness remained. But for others, "the affection is taken over by the pressure of caring" (CSR002).

\section{Chasing the disease}

The progressive nature of MND meant that carers found themselves managing a situation that was never static: "because it never plateaued, it just kept going downwards." (BSH015) They stepped in to compensate for the deterioration in the patient: "You're on a roll, aren't you? [...] You're like a hamster on a wheel, and each day or each week or each month, you do that little bit more and a little bit more" (BSR013). Carers found themselves managing one set of limitations when another deterioration happened: something new to deal with, while also coping with the psychological impact of further deterioration. Speed of progression meant there was an immediacy to patients' needs that was often at odds with time taken to get supportive input in place. (Referring to the need for changes to a bathroom) "we were told we might wait between four and 6 months to be assessed. And then you've got to wait for the work to be done. Well, we needed it doing there and then." (CSH055). Many times they arranged for equipment to be provided at their own expense, so it was in place at the time it was needed.

\section{Intensity of caregiving}

Caregiving experiences were unique, but there was a commonality in terms of the intense nature of their role which in part related to being partner/spouse of the person with MND: someone with whom they had a close personal relationship. A strong sense of responsibility for caregiving was combined with sadness and emotional vulnerability: "because you feel so inadequate, you want to make it better for them, you can't." (CSH055). As MND quickly affected patients' abilities to manage ADLs, carers often became 'hands on' at an early stage. Dependency on the carer was $24 / 7$, including providing care at night, because there was no one else. Complexity of caregiving and constant vigilance required were also factors in this intensity.

\section{(2) Domains of support for carers of people with MND}

Carers spoke in positive terms about support from healthcare professionals, but this was for the patient, less so about separate support for themselves as carers: "Individually, they've not provided that support, because that's not their brief, it's to look after (the patient)" (CSH037). Commonly carers were asked 'are you alright'? "And, of course, you say, yes, you are alright, because you've got to be alright, you've got no option, have you?' (BW002). But others felt 'abandoned' or 'invisible' within patient consultations with healthcare teams, despite having many support needs.

\section{Direct domains: carers' own health and well-being needs}

Carers' discussions revealed the extent of 'direct' support needs: support required to preserve their own health and well-being in their role as 'clients'. Table 4 provides illustrative examples of the range of support needs (both met and unmet) within each broad domain and input required to meet those needs.

Getting a break from caregiving depended on stage of illness. Initially, carers were able to get short periods away but only if patients could be left comfortably and safely, for example, with food/drinks; able to access the toilet. Availability of professional carers varied greatly: some carers only had support from family or paid for private respite. It was much more difficult to leave patients in later-stage MND where symptoms needed constant attention. However, most breaks were to do tasks like shopping or housework rather than actual time for themselves, though carers recognised that it was important to create some separate space for themselves: "It's snatching time" (CSR030).

Being a carer overnight was exhausting: requiring constant vigilance. Carers were aware of limited respite services but lack of discussion by healthcare practitioners about this in itself was difficult. A common dilemma carers faced was of needing a break but having feelings of guilt and ambivalence with regard to having their own needs met. The impact of overnight caregiving on physical health was substantial: "I was rocking with exhaustion" (CSH041). Carers were aware of the effects, but had little help to do anything about it.

With financial, legal and work issues, carers accessed help/advice from many sources, but a recurrent theme was input needed earlier in the illness: proactive or anticipatory advice/information and signposting on. Many carers went through an ad hoc process of discovering benefits/allowances, often missing out on certain entitlements. Need for practical help within the context of MND, extended beyond the home to the garden and to transport issues from the home, including parking, but 
Table 4 'Direct' domains: direct support to carers to preserve their own health and well-being as 'clients'.

Domains of support needs

Having time for yourself in the day

Getting a break from caring overnight health (physical problems)

Key aspects of support identified in interviews/focus groups with carers

Met needs/unmet needs with... Supportive input (received or needed)

patient refusing to have help from anyone other Advice and information:

than carer

managing the patient who is frightened to be alone without the carer even for short periods for example, to visit own GP

dealing with not being able to get out because patient cannot be left

getting away from the 'unfairness' of MND feeling that they should be there and doing things $24 / 7$ particularly if a spouse/partner as well as a carer

thinking it is legitimate to get a break (carers tend not to think about a break for themselves) getting a few hours in the week to do a range of necessary tasks: food shopping, going to bank, going to post office, changing library books, getting housework done, attending appointments

- dealing with healthcare professionals who consider that carers need time, not for self, but only to go to post office, buy food

having some time just for themselves/what they want to do: carers talked about doing something relaxing, being able to unwind, something for their own health/fitness, to go driving as a stress release, going for a coffee, going for a walk, meeting a friend, doing some voluntary work
- about services locally that would provide a break for the carer

Directly delivered input:

- advance booking of short period of respite, for example, through MNDA

- specific breaks from health and care services/ charities: care-team provided via local authority personal budget, professional carers from an early stage to build a relationship with the patient and confidence to be left with them, sitters for some respite hours from charity or from hospice, team providing set hours per week for personal care for the patient

- family help (family events providing a break because more people around to help, direct care help from family members, though carers often reluctant to accept)

- private care teams (at a cost to the patient and carer): agency sitting services; private care team two afternoons a week

Opportunistic breaks:

- when patient attending hospice or day services

- during District Nurse (DN) team visits to the patientpotential cover for the carer to go out

- reliance on friends/neighbours to sit with patient

- by having Macmillan Transport to take patient to hospice appointments

Identified 'downtime' for the carer even if unable to leave the home: for example,

in the late evening when patient is safely in bed

in the early morning before the patient is up

Advice and information:

being up several times during the night because caring involves helping with toileting, managing falls, turning the patient in bed, listening out for the patient

- difficulty of raising need for a break in front of the patient

- feelings about respite (eg, guilt about wanting respite, ambivalence/ reluctance to leave patient, knowing that patient prefers carer to do overnight care, having night respite available but patient not wanting it)

- being able to 'let go' when care worker is providing respite

availability of respite services

Directly delivered input:

night care in the patient's own home (arranged by Macmillan, care worker from the hospice, by family members/shared care overnight, by private arrangement)

- patient admission for a period of respite: to hospital or hospice

Signposting/referral to:

- joint patient and carer break at a respite centre where patient needs met by centre staff overnight as well as in the daytime

- a holiday break with time in the day for the carer to catch up on sleep

Directly delivered input:

- someone to look after patient to give carer time to do exercise/go for a walk

overnight are: fatigue and tiredness due to lack of sleep; weight loss

direct impact of lifting patients: back problems, bad shoulder, hernias

- understanding the impact of caring on carer from the start

- knowing who to talk to about physical effects from the stress of caring role

carer's own health problems: high blood

pressure, illnesses/injuries/symptoms experienced

loss/lack of time for physical exercise

tiredness from doing both caring and working
- a person to look after patient to allow carer to go to hospital for treatment

- physical therapy sessions delivered in the home as carer unable to leave the patient for time to attend clinic

- prescribed medication for health problems

- strengthening exercises at a gym to help with lifting the patient when he falls (because no other help offered)

(Little advice on carers' own health) 
Table 4 Continued

\section{Domains of support needs}

Your financial, legal or work issues

\section{Practical help in the home or elsewhere}

Dealing with your feelings and worries fitting in all the household tasks while caring including washing, ironing, cleaning, shopping, preparing meals

- garden work as patient becomes less able to do it

practicalities of getting to hospital appointments

- patient's refusal to have anyone in the home to help the carer

- cost of having a cleaner to provide some help in the home accepting help offered/provided

- carers' own specific feelings and worries: (eg guilt-if carer gets irritable with the patient or for wanting help for self as a carer when the patient has the illness, having to put on a 'front' of coping because the patient needs to see carer as dealing with things, anxiety about new symptoms of progression of the illness, fear of what lies ahead with the illness, sadness at patient's deterioration, isolation and mental health issues, grieving which began at diagnosis, worry about becoming ill themselves while caregiving)

- patients' reaction to the illness which impacts carers' own mental health (eg, patient not wanting to tell family how he is-carer has the load on his/her own, denial by the patient, too much openness by the patient in discussions about dying causing carer distress)

knowing who to go to for help with feelings

Your beliefs and spiritual concerns dealing with the effect of disease on personal beliefs, including challenges to those beliefs - understanding issues and feelings around assisted dying

\section{Directly delivered input:}

someone to talk to (soon after diagnosis, from the medical team to talk with the carer alone about how they were managing MND as a couple, at a regular appointment following referral-an hour of talking, in the middle of the night when frightened-a helpline, someone to call the carer regularly - to just listen)

(Range of people provide this support: family members, a network/circle of friends, friends in the church, from MNDA carers' meeting to talk openly, away from the patient)

Directly delivered input (in addition to talking):

- getting out to do gym sessions

- medications for anxiety/depression

Signposting/referral:

to more specific mental health input where needed

DN, district nurse; GP, general practitioner; HCP, health care professional; MND, motor neurone disease; MNDA, Motor Neurone Disease Association. 
they were rarely asked about this: "actually sometimes it's just for them to say 'No, I can see you're struggling"” (CSH047). Carers often had difficulties accepting help, but this was true across all the support domains, not just practical help.

The emotional impact of caregiving was harder to deal with for some carers, than physical effects, and they didn't always have an outlet for their feelings. They needed support to deal with their own reactions to the illness but also the patient's response. A worry commonly voiced was what would happen if they became ill, or worst-case scenario, they died while caregiving: "If something happens to me, then we're in trouble because I do everything for (patient)" (CSH037). The diagnosis of MND challenged the belief systems of both patients and carers and raised needs about information and discussions about assisted dying.

\section{'Enabling' domains: support needs in caring for the patient}

Carers also had a range of support needs to enable them to care for the person with MND in their role as 'co-workers' (see table 5). They provided an extensive range of support, including assisting with all ADLs. Carers received help from different professional care teams, but these were time-limited visits, leaving carers to manage for the remaining hours. Managing ADLs necessitated not just advice, but 'training": "I had to learn as I was going. along. [...] You need somebody really that could take you to one side and show you how to do it" (BSR003). "Yeah. Well, it's basic things like learning how to lift them up out of the chair or things like that, or help them out of bed, to roll over and that kind of thing" (BSR017).

Carers needed to know about, access and be able to use many different pieces of equipment to manage ADLs. Although equipment was for the patient, carers were clear that it supported them in caregiving: "I don't need support particularly for me, but I do need equipment to help me do what $I d o "$ (CSH045) and they also needed training to use this equipment. Dealing with MND symptoms involved managing complex medical devices in addition to medicines, again requiring advice/information, but importantly training in their use. Some found this worrying, others were fine: "Once I got the confidence I was fine and it suited (partner) because she didn't want any help [...] so it was just me and her right until the end really" (BW009). As a result, carers became expert in managing patients' needs.

Carers needed to be able to contact services if concerned and at its most basic that meant 24 hours phone services. It was also about having a key contact person, and different professionals took on this role including occupational therapists, community matrons, district nurses, general practitioners (GPs) and MND specialist nurses. However, in the context of MND, carers were very concerned about patients' ability to summon help if carers themselves became ill, identifying the importance of a contact to check on carers of patients in the later stages of MND.

Support needs in understanding the illness were time related: "I don't particularly need any more information at the moment about understanding my relative's illness, but I would have done (earlier)" (CSH014), particularly around diagnosis. General information was needed then but also someone with knowledge of MND to answer specific questions. Talking with their relative about his/her illness was difficult for many carers, needing support with managing issues of denial from both sides and for some also suggestions of suicide. Carers also experienced considerable difficulties in accessing any support for themselves when patients refused to talk about their illness or let anyone know about the diagnosis.

Regarding knowing what to expect in the future, some carers preferred not to know, living each day at a time, though they also acknowledged that 'not knowing' was hard. Where carers wanted this support, they found some healthcare professionals reluctant to talk about dying: "vague talk" (BW003D) wasn't helpful in making preparations for the further decline and death.

\section{(3) An additional domain of support needs in MND}

Stage 1 FGs and interviews identified that support needs in MND mapped well to the existing 14 CSNAT domains and this was later confirmed by carer advisors in Stage 2 workshops. These workshops also sought to identify any aspects that didn't map or suggested missing domains. One aspect of caregiving, dealing with relationship changes as a result of MND, was further explored to determine whether support needs arising from these changes were encompassed by existing CSNAT domains or an additional, separate, domain was needed.

MND affected relationships in different ways for different people. Some felt that difficulties were related to frustrations from the loss of control and role changes patients experienced, and this was difficult to talk about. Relationship issues could be part of the CSNAT 'feelings and worries' domain, but depended on circumstances. An alternative domain was 'talking to your relative about his/ her illness', though this could be perceived as having a narrower, physical focus: "As I say, I think the physical things sometimes are easy [...], but it's the mental thing with your relationship and everything" (CSH034). Overall, the consensus was that it was important to add a separate domain about relationships, one that was more specific: “...because, while yes, it does fit into these two categories really well, but then it's that, happy to verbalise it, which is sometimes the hardest part isn't it? Getting people to say, this is actually what's bothering $m e "$ (CSH047).

Carers identified several reasons for having a separate relationship domain. It could prepare new carers for something that might affect them in the future. Just as carers may not have support needs within some of the CSNAT domains in the early stages but these arise later, so too with the relationship domain. Changes in relationships usually evolved over the course of the illness, and were not necessarily present at the start. What was important to carers was that there was a choice in being able to discuss support with relationships issues, should they arise. Recognising the conservative nature of most people about talking about relationship changes such as 
Table 5 'Enabling' domains: support for carer to care for the patient in their role as 'co-workers'

\section{Domains of} support needs

Providing personal care for your relative

Equipment to help care for your relative
Key aspects of support identified in the interviews/focus groups with carers

Met needs/unmet needs with... Supportive input (received or needed)

managing/helping patient with ADLs: (getting Advice and information:

up in the morning/to bed at night, dressing anticipatory guidance on how to manage ADLs and undressing, washing/bathing/showering, proactive advice on getting carer team input with toileting - both in the day and at night, managing incontinence, dealing with soiling, managing catheters, all aspects of mobility: lifting or moving including in bed, managing patient falls, feeding the patient, including avoiding loss of weight)

- understanding changes in mobility/ movement as disease progresses

strain of being the only person the patient permits to help with ADLs

being able to give carer perspective when patient is not being fully honest about how he/she is managing managing the cost of paying for private carers

personal care and how to access care services

- on completing forms for continuing healthcare

- from continence service

- practical tips for managing outside the home,for example, how to access a radar key for disabled toilets

Education/training - needed from 'day one':

- lifting and handling

- how to do a bedbath; washing/cleansing to deal with incontinence and soiling

- hygiene requirements for managing catheters

- individualised dietary advice appropriate to the carer's situation

Directly delivered input:

- provision of equipment by different agencies (local councils, MNDA) and professionals (such as OTs) enabling carers to provide personal care, for example, sliding boards, hoists, commodes, and so on

- help from professional care team with showering and getting patient up/to bed but requires continuity and reliable timing

- private care assistants to do personal care

- care packages from continuing healthcare

- DN assistance with changing catheters

- regular contact from DN team to see how carer was managing

- help from neighbours when patient falls

- help from ambulance service with lifting

- short-term 'emergency' care team four times/day for 1 week on leaving hospital

Advice and information:

anticipatory guidance from HCPs on types of equipment likely to be needed during the illness equipment to help manage the patient's illness

- accessing specific pieces of equipment/ aids including walking aids, seat raisers, wheelchairs, commodes, shower stools, perching stools, manger air cushions, fold up chairs that goes in car, hoists, hospital beds, special cups, special cutlery, zimmers, walking trolleys, walking sticks, hand rails, boogie board, iPads with predictive text.

- making adaptations to the home to help with managing the needs of the person with MND: including putting in showers, wet rooms, raised toilets, full lifts, stair lifts, outside ramps

- managing cost implications of paying for equipment/adaptations to respond to immediacy of the patients' needs
- agencies providing different equipment (locally): therapy services, local councils, MNDA

- website for ordering equipment accessible by carers

- MNDA grants to help with the cost of equipment

Education/training in use of a range of equipment:

- such as hoists, sliding mats

Directly delivered input:

- timely referral by MND nurse to Occupational Therapist (OT) at local council for input

- a named OT visiting regularly to review equipment needed

- services taking account of patient/carer preferences in equipment provided

- equipment actually wanted: for example, a hospital bed may not be a supportive input for all carers

- equipment actually needed: for example, iPad may not help when family already has one 
Table 5 Continued

\section{Domains of support needs}

Managing your relative's symptoms including giving medicines

Key aspects of support identified in the interviews/focus groups with carers Met needs/unmet needs with... Supportive input (received or needed)

managing patient symptoms: (difficulties swallowing, choking, excess secretions/ saliva, breathing difficulties/shallow breathing, panic attacks, terminal agitation in the end stages)

using different appliances to manage symptoms including Cough Assist, suction, respirators, PEG or RIG tubes

dealing with responsibility for managing RIG feeling helpless during a choking episode managing reluctance of patient to take drugs to help with panic attacks administering medicines down the feeding tube

accessing specialist nutrition for patient each month

Advice and information:

- how to manage a choking episode

- breathing problems in an emergency from ambulance service/paramedics

- how to handle better a panic attack

- managing communication difficulties

- contacting the feeding company if any problems

Education/training:

- managing PEG/RIG including using it to provide patient's nutrition, cleaning it/preventing infection, clearing any blocking of the tube

- fitting of a feeding tube prior to start of choking episodes

- managing the patient's respirator

- using Cough Assist

Directly delivered input:

- provision of oxygen in the home

- having an efficient delivery system of specialist nutrition so that correct prescription is supplied

- initial supervision of carer managing PEG/RIG, including when the patient returned home

- GP help in getting medication in liquid form

- drugs to assist carer dealing with patient panic attacks

- local administration of Botox injections to dry up saliva rather than a 5 -hour round trip to main hospital

- setting up syringe driver to settle patient at end stage

Knowing who to contact if you are concerned about your relative
- confusion over which professional does what Advice and information: and which part of the NHS they are from - ensuring correct details for night-time contacts

- dealing with changes that occur and help that is needed

- accessing MND expertise in an emergency situation

- potential situation of carer becoming ill/has an accident/dying and patient being unable to raise alarm message) who does what a care team to come

- Most basic-a contact number (available 24/7, not just office hours, in primary care/GP surgery, if an answer-machine-a timely response to the

- a'contacts' book - of numbers of HCPs including

- An emergency contact for example,'Carers First' - provides a number the patient can ring if something happens to the carer and they organise

Having responsive contact:

a person to talk to/have a conversation (who understands the caring situation in MND; who knows how to access help; to visit at home to facilitate further support and provide continuity) Proactive contacts:

at regular times along the caring journey

- a checking system in late stages of MND to ensure carer is alright

Talking to your relative about his/ her illness dealing with the patient's reaction to the diagnosis,for example, denial, threats of suicide

patient's refusal to let people know about the illness

patient's refusal to talk about their (joint) situation of living with the disease

understanding the patient's situation/ mental well-being separate from the clinical condition

being able to discuss with the patient, the carer's role in providing care

- with carer's own denial of the diagnosis
Directly delivered input:

an opportunity to talk about their situation as a carer

- regular visit by MND nurse just to talk with patient and carer about their situation

Referral:

to a counselling/support group for patient and carer

- of the patient for counselling (was a support for the carer) 
Table 5 Continued

\begin{tabular}{lr} 
Domains of $\begin{array}{c}\text { Key aspects of support identified } \\
\text { support needs }\end{array}$ & \multicolumn{1}{c}{ Met needs/unmet needs with... } \\
\cline { 2 - 2 } $\begin{array}{l}\text { Understanding your relative's } \\
\text { illness }\end{array}$ & $-\begin{array}{l}\text { understanding the different stages of the } \\
\text { illness including which stage the patient is } \\
\text { currently at }\end{array}$ \\
- & understanding the speed of progression of \\
& the illness \\
- & knowing the restrictions of the disease
\end{tabular}

Key aspects of support identified in the interviews/focus groups with carers support needs

Understanding the different stages of the currently at the illness

nowing the restrictions of the disease

Advice and information:

initial general information about MND (usually from MNDA)

Directly delivered input:

- an early (proactive) contact by healthcare professional for discussion following shock of the diagnosis

- consultations with a person who understands MND to answer questions: specialist nurses, GPs, community matrons

- a separate explanation to the carer about the disease they are dealing with to sensitise them to the changes

- carer/consultant consultation to ask questions without patient present

\begin{tabular}{|c|c|c|}
\hline $\begin{array}{l}\text { Knowing what to expect in the } \\
\text { future }\end{array}$ & $\begin{array}{l}\text { - } \text { fears/worries about managing next stage of } \\
\text { deterioration } \\
\text { - } \quad \text { ambivalence of wanting to know about the } \\
\text { future } \\
\text { - } \quad \text { talking about the dying process } \\
-\quad \text { preferred place of care discussions } \\
-\quad \text { treatment decisions (do not resuscitate } \\
\text { (DNR) - with patient and carer and their } \\
\text { situation as a couple, patients' decision on } \\
\text { DNR/or not, refusing treatment, respect from } \\
\text { hospital about DNR signed by the patient) } \\
-\quad \text { dealing with the unpredictability of prognosis } \\
-\quad \text { understanding the proximity of death } \\
-\quad \text { issues arising after the death (moving the } \\
\text { body after death, funeral arrangements) }\end{array}$ & $\begin{array}{l}\text { Advice and information: } \\
\text { symptoms to expect as patient deteriorates } \\
\text { illness trajectory (some relied on discussion } \\
\text { of patient symptoms in clinics as a clue to } \\
\text { progression) } \\
\text { realistic prognosis including preparing for a short } \\
\text { prognosis } \\
\text { signs of dying } \\
\text { services providing support like hospice at home } \\
\text { Directly delivered input - pro-actively: } \\
\text { revisiting what to expect over the course of the } \\
\text { illness, not just a one off. } \\
\text { advance care planning discussions to put suppor } \\
\text { in place when needed } \\
\text { DNR and advance refusal of treatment } \\
\text { discussions as part of care from GP } \\
\text { from OT service on equipment likely to be needeo } \\
\text { visits from the carers' centre to discuss 'what the } \\
\text { future holds' } \\
\text { Openness by HCPs to talk when family ask: } \\
\text { honesty about what death involves } \\
\text { that time of death is close so family can prepare } \\
\text { and be present }\end{array}$ \\
\hline
\end{tabular}

ADL, activities of daily living; DN, district nurse; DNR, do not resuscitate; GP, general practitioner; HCP, health care professional; NHS, National Health Service; OT, occupational therapist; PEG, percutaneous endoscopic gastrostomy; RIG, radiologically inserted gastrostomy.

intimacy, a separate domain was felt to give "permission to talk about something very private" (CSH055), if they wished to.

Workshop discussions further revealed that carers' support needs with relationship issues extended beyond spousal relationships: "there's all sorts of relationship groups that are affected because of the illness. Friendship groups, work colleagues, social groups. Relationships with healthcare professionals as well, there might be a conflict with who your current healthcare professionals are. [...] So perhaps having that extra domain that actually bring out some of those issues" (CSR048). Wording of the domain thus needed to reflect support needs within more wide-ranging relationships. Different options were explored initially in the workshops, with email and telephone follow-up iterations. 'Do you need more support with managing relationships' was finally agreed and added to the existing CSNAT questions to be piloted in Stage 3 of the study (to be reported elsewhere).

\section{DISCUSSION}

This paper examines experiences of caregiving in the context of MND. Carers' lives were significantly impacted by the disease. Study findings suggest that adapting the existing CSNAT through the addition of a new domain on support needs with relationship changes will enable identification of the wide range of support needs experienced by carers of people with MND.

Overall, support needs in MND caregiving mapped well to existing 'enabling' and 'direct' CSNAT domains and carers found the domains appropriate and relevant: a finding supported by a pilot study using the CSNAT intervention in the context of MND in Australia. ${ }^{29}$ However, our in-depth exploration of carers' support needs also identified that a further assessment domain was required to address role and relationship changes due to MND, commonly reported aspects of the experience of MND 
caregiving. ${ }^{4}{ }^{30-32}$ However, the need for such an additional domain may not be required for MND per se, but may be reflective of support needs arising from prolonged intensive caregiving. Farquhar et $a l^{33}$ reported similar role changes experienced by carers of patients with breathlessness in advanced chronic obstructive pulmonary disease (COPD). More recently, two systematic reviews of support needs of carers of patients with $\mathrm{COPD}^{34}$ and of people living with pulmonary fibrosis and their caregivers ${ }^{35}$ identified similar difficulties within patient-carer relationships. The COPD review also identified support needs with carer-clinician relationships, recommending an additional CSNAT domain to encompass the full range of support needs of these carers. ${ }^{34}$ The original study to develop $\operatorname{CSNAT}^{19}$ mainly involved carers in a cancer context where intensive caregiving was much shorter term. It furthermore included only bereaved carers, many of whom reflected back on the uncomplaining nature of those they cared for and not on the tensions expressed in the current study.

The extent of carers' support needs in MND in this study evidences the necessity of a separate process of assessment and support for MND carers. Carers furthermore required support to enable them to support the patient as 'co-workers' and direct support to look after their own health and well-being as 'clients'. Current guidance, such as from the National Institute for Health and Care Excellence (NICE) ${ }^{36}$ recommends advising carers of their legal right to a Carer's Assessment but this fails to take account of this dual role carers play and their support needs in both roles. While some needs for carers as 'clients' may be addressed by the statutory carer assessment, these assessments do not identify the needs carers have as 'co-workers', where they rather need healthcare professional input to enable them to provide care for the person with MND. The extent of support needs within these 'enabling' domains and the burden carers experience from caregiving evidences a need for a more comprehensive assessment process. The broad domains of the CSNAT are intended to help open conversations with carers by providing visibility about aspects of support others in their situation have found helpful. Which individual needs are discussed within domains depends on how those domains resonate with individual carers: what is key is that they facilitate a conversation to uncover the carer's individual needs which can then be supported.

While there is a wide literature on carers' needs in MND, a strength of this study is that our findings specify in detail many different types of support carers needed or found helpful from healthcare/social care professionals. 'Pro-active' input was identified as particularly important across many domains, that is guidance ahead of need, not just 'reactive' input to a problem or crisis, which resonates with findings from a meta-analysis of carers' educational needs. ${ }^{37}$ Certain types of input that may be delivered directly by professionals were common across domains: particularly advice and information (ranging from very general to highly tailored); training in different care activities; or directly delivered help. Family and friends may also provide some direct help. However, some support needs may necessitate signposting and referral by healthcare/social care professionals to other support agencies. These common themes and detailed analyses of needs experienced offer practical guidance to assist practitioners in ensuring help is tailored to carers' individual needs.

\section{Limitations of the study}

This study was qualitative with a self-selecting sample, so findings may not be fully generalisable. However, the three study sites where recruitment took place had very different MND management protocols which adds validity in terms of transferability of findings to other centres and practitioners working with patients with MND and their carers. We also believe that the findings will have relevance for practitioners and carers managing all stages of the illness as we were able to conduct interviews with carers from throughout the illness trajectory from newly diagnosed MND to advanced disease and into bereavement.

\section{Implications for practice}

In the first two stages of this study an adapted version of the CSNAT, comprising the existing 14 domains plus a new domain on support with managing relationships was developed for implementation as part of a practice intervention for MND carers (Stage 3 study findings to be reported elsewhere). Carers found the adapted CSNAT to be an appropriate and relevant tool for assessment of their support needs. The revised version also has potential for assessment of carers in other longer-term caring contexts. Furthermore, the detailed exploration of the input carers themselves have identified as important in meeting their different support needs provides a valuable training resource to assist practitioners in tailoring support provision to carers in the context of MND.

Twitter Gail Ewing @gailewing_cfr and Christine Rowland @c_a_rowland

Acknowledgements The authors thank the carers and carer advisors who took part in this study for their time and contribution, and members of the Salford MNDA and Study Advisory Group. The authors also thank the teams supporting MND carers at Sheffield Teaching Hospitals NHS Foundation Trust, Salford Royal NHS Foundation Trust and The Walton Centre NHS Foundation Trust for their help with the study.

Contributors GE: co-lead, designed the study, acquisition of funding, data collection, analysis and interpretation of data, drafted the article; SC: data collection, interpretation of data and revised article critically for important intellectual content; CR: designed the study, data collection, analysis and interpretation of data and revised the article critically for important intellectual content; GG: project lead, designed the study, acquisition of funding, data collection, analysis and interpretation of data and revised the article critically for important intellectual content.

Funding The study was funded jointly by the Motor Neurone Disease Association and Marie Curie: Marie Curie Project Award - Reference number MCRGS-07-1621. The work was supported by NIHR CLAHRC Greater Manchester. The views expressed in this article are those of the author(s) and not necessarily those of the NHS, NIHR or the Department of Health.

Competing interests None declared. 
Patient and public involvement Patients and/or the public were involved in the design, or conduct, or reporting, or dissemination plans of this research. Refer to the Methods section for further details.

Patient consent for publication Not required.

Ethics approval Ethics approval was received from the North West - Greater Manchester West Research Ethics Committee (REC reference: 17/NW/0531).

Provenance and peer review Not commissioned; externally peer reviewed.

Data availability statement All data relevant to the study are included in the article or uploaded as supplemental information. No additional data are available.

Open access This is an open access article distributed in accordance with the Creative Commons Attribution Non Commercial (CC BY-NC 4.0) license, which permits others to distribute, remix, adapt, build upon this work non-commercially, and license their derivative works on different terms, provided the original work is properly cited, appropriate credit is given, any changes made indicated, and the use is non-commercial. See: http://creativecommons.org/licenses/by-nc/4.0/.

Accessing the CSNAT The CSNAT is a copyright tool available free of charge to the National Health Service (NHS) and not for profit organisations. Training and a licence are required for its use as a practice intervention. For further details go to http://csnat.org.

ORCID iD

Gail Ewing http://orcid.org/0000-0001-9547-7247

\section{REFERENCES}

1 MNDA. Key facts and information about MND. Available: https:// www.mndassociation.org/about-us/who-we-are/mnd-key-facts/ [Accessed 17 Feb 2020].

2 MND Australia. Facts and figures. Available: https://www.mndaust. asn.au/Get-informed/What-is-MND/Facts-and-figures.aspx [Accessed 17 Feb 2020].

3 ALS Association. Who gets ALS? Available: http://web.alsa.org/site/ PageServer?pagename=ALSA_WhoGets [Accessed 17 Feb 2020].

4 Aoun SM, Bentley B, Funk L, et al. A 10-year literature review of family caregiving for motor neurone disease: moving from caregiver burden studies to palliative care interventions. Palliat Med 2013;27:437-46.

5 de Wit J, Bakker LA, van Groenestijn AC, et al. Caregiver burden in amyotrophic lateral sclerosis: a systematic review. Palliat Med 2018;32:231-45.

6 Galvin M, Corr B, Madden C, et al. Caregiving in ALS - a mixed methods approach to the study of Burden. BMC Palliat Care 2016;15:81.

7 Goldstein LH, Atkins L, Landau S, et al. Predictors of psychological distress in carers of people with amyotrophic lateral sclerosis: a longitudinal study. Psychol Med 2006;36:865-75.

8 Pagnini F, Rossi G, Lunetta C, et al. Burden, depression, and anxiety in caregivers of people with amyotrophic lateral sclerosis. Psychol Health Med 2010;15:685-93.

9 Peters M, Fitzpatrick R, Doll H, et al. The impact of perceived lack of support provided by health and social care services to caregivers of people with motor neuron disease. Amyotroph Lateral Scler 2012;13:223-8.

10 Whitehead B, O'Brien MR, Jack BA, et al. Experiences of dying, death and bereavement in motor neurone disease: a qualitative study. Palliat Med 2012;26:368-78.

11 Creemers $\mathrm{H}$, de Morée $\mathrm{S}$, Veldink JH, et al. Factors related to caregiver strain in ALS: a longitudinal study. J Neurol Neurosurg Psychiatry 2016;87:775-81.

12 Lorenz KA, Lynn J, Dy SM, et al. Evidence for improving palliative care at the end of life: a systematic review. Ann Intern Med 2008;148:147-59.

13 Candy B, Jones L, Drake R, et al. Interventions for supporting informal caregivers of patients in the terminal phase of a disease. Cochrane Database Syst Rev 2 011;15:CD007617.

14 Gomes B, Calanzani N, Curiale V, et al. Effectiveness and costeffectiveness of home palliative care services for adults with advanced illness and their caregivers. Cochrane Database Syst Rev 2013b;6:CD007760.
15 Harris M, Thomas G, Thomas M, et al. Supporting wellbeing in motor neurone disease for patients, carers, social networks, and health professionals: a scoping review and synthesis. Palliat Support Care 2018;16:228-37.

16 Bentley B, O'Connor M, Breen LJ, et al. Feasibility, acceptability and potential effectiveness of dignity therapy for family carers of people with motor neurone disease. BMC Palliat Care 2014;13:12-22.

17 Aoun SM, Chochinov HM, Kristjanson LJ. Dignity therapy for people with motor neuron disease and their family caregivers: a feasibility study. J Palliat Med 2015;18:31-7.

18 O'Brien MR, Jack BA, Kinloch K, et al. The Carers' Alert Thermometer (CAT): supporting family carers of people living with motor neurone disease. Br J Neurosci Nurs 2019;15:114-24.

19 Ewing G, Grande G. Development of a carer support needs assessment tool (CSNAT) for end-of-life care practice at home: a qualitative study. Palliat Med 2013;27:244-56.

20 Ewing G, Austin L, Diffin J, et al. Developing a person-centred approach to carer assessment and support. Br J Community Nurs 2015;20:580-4.

21 Ewing G, Austin L, Grande G. The role of the carer support needs assessment tool in palliative home care: a qualitative study of practitioners' perspectives of its impact and mechanisms of action. Palliat Med 2016;30:392-400.

22 Ewing G, Brundle C, Payne S, et al. The carer support needs assessment tool (CSNAT) for use in palliative and end-of-life care at home: a validation study. J Pain Symptom Manage 2013;46:395-405.

23 Aoun S, Deas K, Toye C, et al. Supporting family caregivers to identify their own needs in end-of-life care: qualitative findings from a stepped wedge cluster trial. Palliat Med 2015;29:508-17.

24 Aoun S, Toye C, Deas K, et al. Enabling a family caregiver-led assessment of support needs in home-based palliative care: potential translation into practice. Palliat Med 2015;29:929-38.

25 Aoun SM, Grande G, Howting D, et al. The impact of the carer support needs assessment tool (CSNAT) in community palliative care using a stepped wedge cluster trial. PLoS One 2015;10:e0123012.

26 Grande GE, Austin L, Ewing G, et al. Assessing the impact of a carer support needs assessment tool (CSNAT) intervention in palliative home care: a stepped wedge cluster trial. BMJ Support Palliat Care 2017;7:326-34

27 Morgan DL. Focus groups as qualitative research. Thousand Oaks: Sage Publications Inc., 1997.

28 Hsieh H-F, Shannon SE. Three approaches to qualitative content analysis. Qual Health Res 2005;15:1277-88.

29 Aoun SM, Deas K, Kristjanson LJ, et al. Identifying and addressing the support needs of family caregivers of people with motor neurone disease using the carer support needs assessment tool. Palliat Support Care 2017;15:32-43.

30 Aoun SM, Connors SL, Priddis L, et al. Motor neurone disease family carers' experiences of caring, palliative care and bereavement: an exploratory qualitative study. Palliat Med 2012;26:842-50.

31 Gluyas C, Mathers S, Hennessy Anderson N, et al. Factors to consider for motor neurone disease carer intervention research: a narrative literature review. Palliat Support Care 2017;15:600-8.

32 Holkham L, Soundy A. The experience of informal caregivers of patients with motor neurone disease: a thematic synthesis. Palliat Support Care 2018;16:487-96.

33 Farquhar M, Higginson IJ, Booth S. Diversity of experiences and impacts of caring for a patient with breathlessness in advanced COPD. Palliat Med 2010;24:211.

34 Micklewright K, Farquhar M. Does the carer support needs assessment tool cover the established support needs of carers of patients with chronic obstructive pulmonary disease? A systematic literature search and narrative review. Palliat Med 2020:269216320939243.

35 Lee JYT, Tikellis G, Corte TJ, et al. The supportive care needs of people living with pulmonary fibrosis and their caregivers: a systematic review. Eur Respir Rev 2020;29:190125.

36 National Institute for Health and Care Excellence. Motor neurone disease: assessment and management. NICE guideline, 2016. Available: https://www.nice.org.uk/guidance/ng42/resources/ motor-neurone-disease-assessment-and-management-pdf1837449470149[Accessed 20 Feb 2020].

37 Flemming K, Atkin K, Ward C, et al. Adult family carers' perceptions of their educational needs when providing end-of-life care: a systematic review of qualitative research. AMRC Open Res 2019;1:2. 